\title{
Digital Artery Massage for Improving Ischemia after Distal Digital Replantation Surgery
}

\author{
Yosuke Niimi, MD, $\mathrm{PhD}^{1, *}$ Hiroshi Ito, $\mathrm{MD}, \mathrm{PhD}^{2, *}$ \\ Junji Hishiyama, MD ${ }^{1}$ Hiroyuki Sakurai, MD, PhD ${ }^{1}$ \\ ${ }^{1}$ Department of Plastic and Reconstructive Surgery, Tokyo Women's \\ Medical University, Shinjuku-ku, Tokyo, Japan \\ 2 Plastic and Reconstructive Surgery Division, Department of Surgery, \\ University of Miyazaki Faculty of Medicine, Miyazaki-shi, \\ Miyazaki, Japan
}

\author{
Karin Ikeda, MD ${ }^{1}$ Miho Kirita, MD ${ }^{1}$
}

J Reconstr Microsurg Open 2018;3:e25-e27.
Address for correspondence Yosuke Niimi, MD, PhD, Department of Plastic and Reconstructive Surgery, Tokyo Women's Medical University, 8-1 Kawada-cho, Shinjuku-ku, Tokyo 162-8666, Japan (e-mail: niimi.yosuke@twmu.ac.jp).

\begin{abstract}
Keywords

- massaging

- fingertip amputation

- antithrombotic agent

Distal digital replantation is frequently associated with arterial thrombosis and/or spasm, leading ischemia in the replanted tissue. This report introduced a rescue technique for ischemia after distal digital replantation without reanastomosis. Two males, 64 and 51 years old, underwent Ishikawa subzone II finger amputations. Microsurgical replantations with vein grafts were performed. Intraoperatively, heparin and urokinase through intra-arterial infusion were given for one week. At 40 to 48 hours after surgeries, the replanted digits developed ischemia; massaging digital arteries at the proximal phalanx regions with running warm water was immediately initiated and ischemia was improved. In both cases, the replanted tissues were rescued, though a partial necrosis requiring full-thickness skin grafting was found in one case. This massage was easily, safely, and effectively performed without complications and was applicable in cases with ischemia after distal digital replantation, especially where reanastomosis was unfeasible.
\end{abstract}

Microsurgeons face serious problems in distal digital replantation. Frequently, distal artery anastomosis is unable to allow surgeons to perform reanastomosis for ischemia due to arterial thrombus and/or spasm, which are main complications after microsurgical replantation. In those cases, amputated digits were monitored as composite grafting. ${ }^{1}$ There are no reports on methods for rescuing replanted digits without medicines other than reanastomosis for postsurgical ischemia. This report shows a simple rescue technique for ischemia after microsurgical replantation.

\section{Case Presentation}

\section{Case 1}

A 64-year-old male with hypertension caught his left middle finger in a shredder and sustained an Ishikawa subzone II $^{2}$ amputation. Microsurgical replantation was performed at

Yosuke Niimi and Hiroshi Ito contributed equally to this report.
10 hours after injury. Since the length of the terminal artery was insufficient, the artery was anastomosed with the vein graft to bridge the segment without venous anastomosis ( - Fig. 1A). ${ }^{3}$ The fish-mouth incision was made to initiate bleeding. Intraoperatively, 10,000 units heparin and 120,000 units urokinase per day were administered continuously through intra-arterial infusion from the left radial artery. Although the inspection at 6 hours after surgery revealed that the finger color and bleeding from the incision were favorable (-Fig. 1B), at 48 hours, the replanted finger became pale and bleeding from the incision ceased ( - Fig. 2A). Losses of Doppler and pulse oximeter signals were observed. Microsurgical reanastomosis was thought to be impracticable because of the terminal artery anastomosis. Instead, massaging the digital artery at the proximal phalanx region with warm water was immediately initiated. Actually, the patient was asked to gently pinch and massage the skin over the radial and ulnar digital arteries of the damaged finger by his right thumb and index finger with $38^{\circ} \mathrm{C}$ warm running water ( - Fig. 2B), and the reciprocating massage was performed at a received

January 7, 2018 accepted after revision March 5, 2018
DOI https://doi.org/

10.1055/s-0038-1642627. ISSN 2377-0813.
Copyright $\odot 2018$ by Thieme Medical Publishers, Inc., 333 Seventh Avenue, New York, NY 10001, USA. Tel: +1(212) 584-4662.
License terms

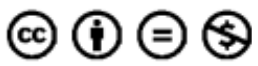




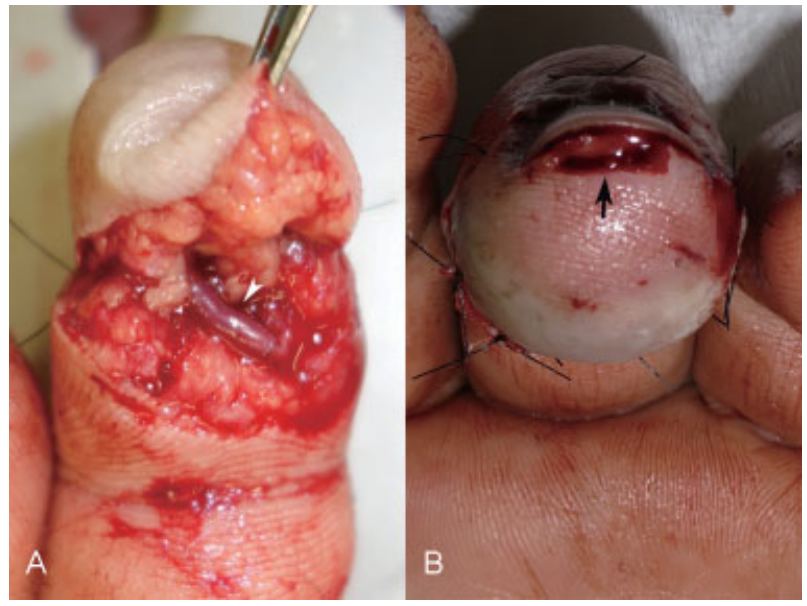

Fig. 1 Intraoperative and postoperative views of the wound site of the middle finger of a 64-year old male. (A) Microsurgical replantation was performed for Ishikawa subzone II amputation of the middle finger. Artery anastomosed with interrupted stitches of 10-0 nylon with the vein graft (arrowhead) to bridge the segment that was harvested from the left medial plantar. (B) At 6 hours after surgery, the examination revealed the color of the replanted finger and bleeding from the fish-mouth incision (arrow) to be favorable.

rate of 100 per minute similar to cardiopulmonary compression. After a 30-minute massage, bleeding from the incision was observed again and the color improved ( $\mathbf{- F i g}$. 2C). Doppler signal was confirmed, and oxygen saturation was measured to be more than $90 \%$ with a pulse oximeter. Thereafter, the digital artery massage once a day with continuous intra-arterial infusion was continued for 1 week. Leech therapy was given when congestion was noted. ${ }^{4}$ The replanted finger was successfully engrafted. At 3 months postoperatively, the morphology of the finger was favorable (Fig. 2D).

\section{Case 2}

A 51-year-old male caught his right thumb in a door and sustained an Ishikawa subzone II amputation. Microsurgical replantation was performed at 8 hours after injury under general anesthesia. Only the artery was anastomosed with the vein graft ( - Fig. 3A). Intraoperatively, heparin and urokinase were continuously given with the same doses as in case 1 . At 24 hours, the finger color and bleeding from the deepithelialized area were favorable ( $\mathbf{F i g}$. 3B). At 40 hours postoperatively, bleeding was ceased and the losses of Doppler and pulse oximeter signals were observed. Because microsurgical reanastomosis was impracticable, massaging digital

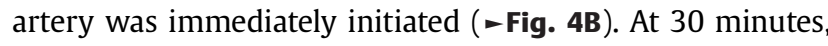
reperfusion was confirmed ( $\mathbf{- \text { Fig. }} \mathbf{4 C}$ ). Doppler signal was confirmed and saturation was observed to be more than $90 \%$. Although partial necrosis was noted, the subcutaneous tissue was engrafted. Only full-thickness skin grafting was performed at 1 month after microsurgical replantation. At 10 months, morphology remains favorable ( - Fig. 4D).

\section{Discussion}

Although composite grafting ${ }^{1}$ or methods by Brent $^{5}$ are occasionally found in cases with postoperative ischemia,

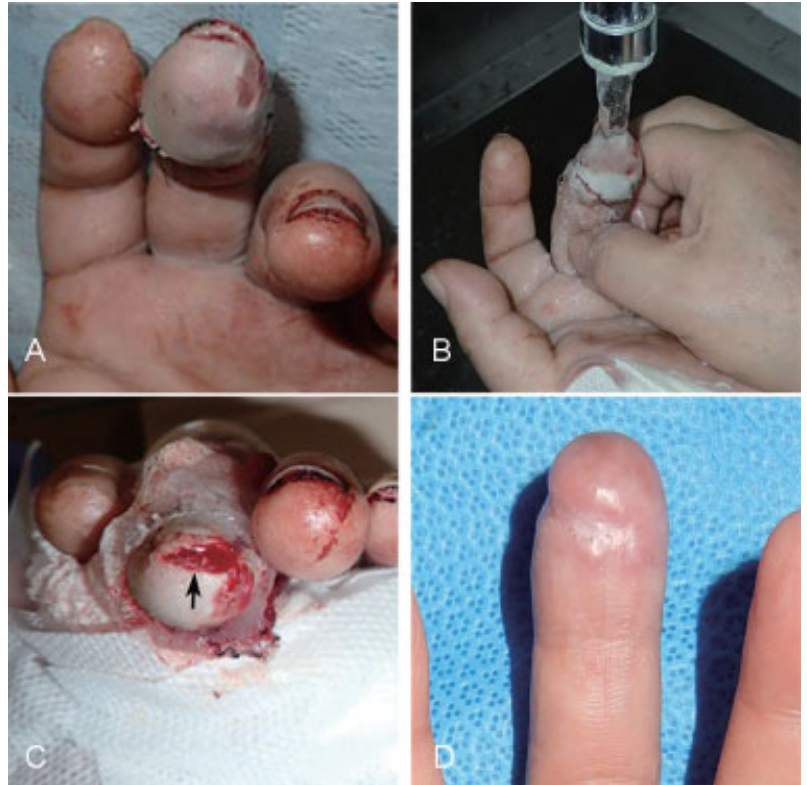

Fig. 2 Postoperative views of the middle finger of a 64-year-old male at 48 hours after surgery. (A) At 48 hours after surgery, the replanted finger became pale, and bleeding from the fish mouth incision ceased, indicating that ischemia appeared at the site due to arterial thrombus. (B) The photograph shows digital artery massage at the proximal phalanx region with running warm water. Massage was performed by the patient's right thumb and index finger at a rate of 100 per minute, similar to cardiopulmonary compression. (C) After a-60 minute massage, bleeding from the fish-mouth incision was observed again, along with reperfusion. (D) At 3 months postoperatively, although mild atrophy was noted, the morphology of the replanted finger was favorable.

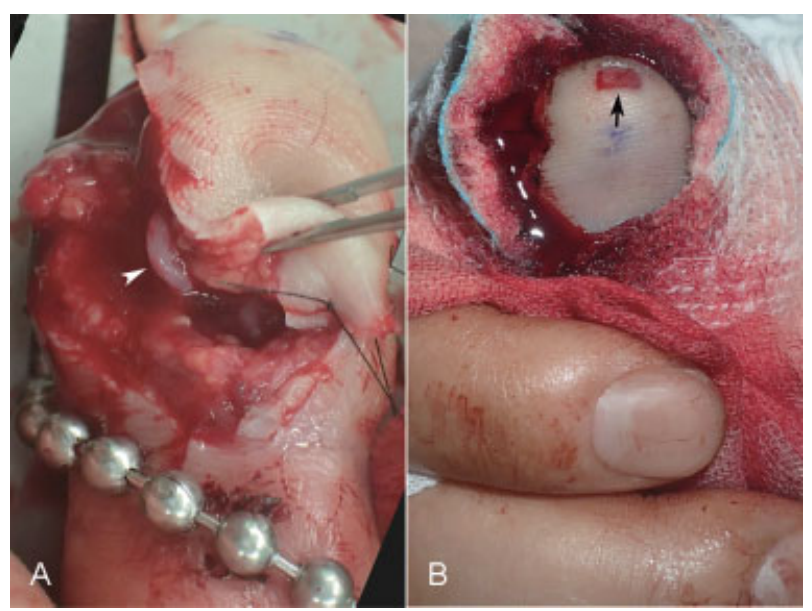

Fig. 3 Intraoperative and postoperative views of the wound site of the right thumb of a 51-year-old male. (A) Microsurgical replantation was performed for Ishikawa subzone II amputation of the right thumb. Artery anastomosed with interrupted stitches of 10-0 nylon with the vein graft (arrow head) to bridge the segment that was harvested from the left medial plantar. The silver ball chain was used for fixing the injured finger whose vessels were anastomosed. (B) At 24 hours after surgery, the examination revealed that the color of the replanted finger and bleeding from the deepithelialized raw surface (arrow) were favorable. 


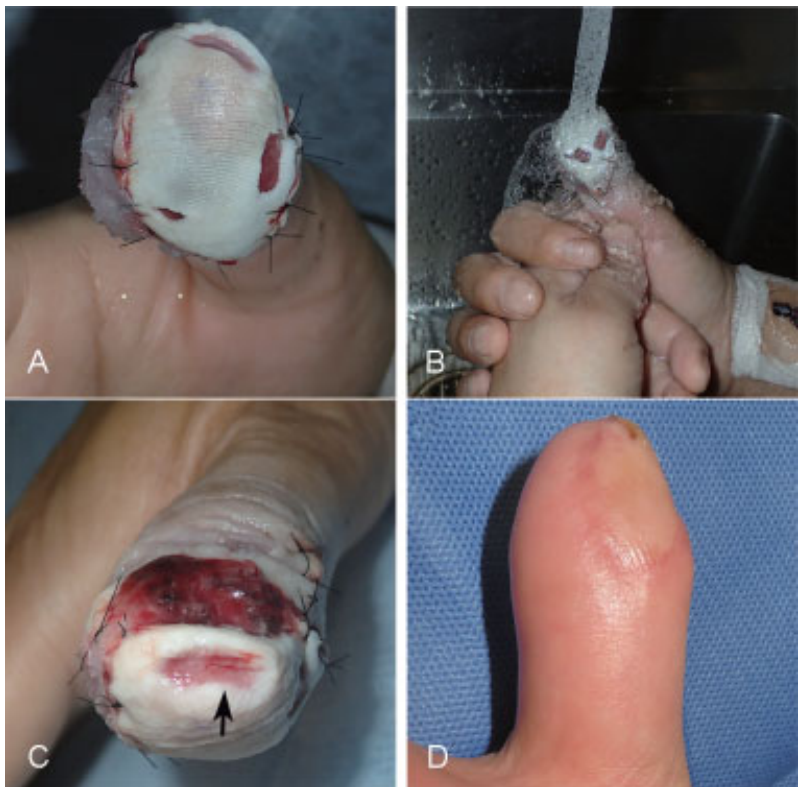

Fig. 4 Postoperative views of the thumb of a 51-year-old male at 40 hours after surgery. (A) At 40 hours after surgery, the replanted finger became pale, the patients felt coldness, and bleeding from the fish-mouth incision ceased, indicating that ischemia appeared because of arterial thrombus. (B) The photograph shows digital artery massage at the proximal phalanx region with running warm water. (C) After 60 minutes of continuing massage, bleeding from deepithelialized area and reperfusion were observed (arrow).

(D) At 11 months after microsurgical replantation, although partial necrosis at the replanted finger was noted, most of the subcutaneous tissue was engrafted. Only full-thickness skin grafting was performed at 1 month after microsurgical replantation.

lower success rates may still remain. ${ }^{1,6}$ In addition, antithrombotic therapy ${ }^{7,8}$ and/or thermal therapy are known to be effective to prevent arterial thrombosis and/or spasm. ${ }^{4}$ However these therapies are insufficient to remove them. In spite of the efficacy of tissue plasminogen activators, ${ }^{9}$ this therapy might have some adverse effects such as bleeding from wound and allergic reactions. The method shown in this report could be performed more easily and safely without any immediate adverse effect.

Advantages of digital artery massage were as follows: (1) an increase in blood flow to the replanted finger by the pumping action of massage as well as artery dilation with warm water, (2) effective thrombectomy by the physical pressure of massage, and (3) thromboprophylaxis and lysis by perfusing pharmacological agents.

This massage was applicable to the following cases: (1) cases where ischemia was found to be distal to subzone III after microsurgical replantation and arterial reanastomosis was found to be impracticable, (2) cases where reperfusion was confirmed after microsurgical replantation, (3) cases where ischemia period was less than 12 hours due to the death of the replanted tissue, ${ }^{10}$ and (4) cases where no anemia was found. Whenever arterial reanastomosis is able to be accomplished, surgeons should perform reanastomosis.

The authors will accumulate cases and evaluate the recovery rate with or without this massage in multiple replantation centers.

\section{Conclusion}

Two cases successfully treated with digital artery massage for curing ischemia after digital replantation were reported. This method would be effective in cases where reanastomosis was deemed unfeasible without composite graft.

\section{Ethical Approval}

This study was performed in accordance with the World Medical Association Declaration of Helsinki (June 1964) and subsequent amendments. The patients voluntarily gave written informed consent to participate in this study.

\section{Conflict of Interest}

None.

\section{References}

1 Moiemen NS, Elliot D. Composite graft replacement of digital tips. 2. A study in children. J Hand Surg [Br] 1997;22(03):346-352

2 Ishikawa K, Ogawa Y, Soeda H, Yoshida Y. A new classification of the amputation level for the distal part of the fingers. J Jpn Soc Microsurg 1990;3:54-62

3 Ito H, Sasaki K, Morioka K, Nozaki M. Fingertip amputation salvage on arterial anastomosis alone: an investigation of its limitations. Ann Plast Surg 2010;65(03):302-305

4 Jazayeri L, Klausner JQ Chang J. Distal digital replantation. Plast Reconstr Surg 2013;132(05):1207-1217

5 Brent B. Replantation of amputated distal phalangeal parts of fingers without vascular anastomoses, using subcutaneous pockets. Plast Reconstr Surg 1979;63(01):1-8

6 Kim KS, Eo SR, Kim DY, Lee SY, Cho BH. A new strategy of fingertip reattachment: sequential use of microsurgical technique and pocketing of composite graft. Plast Reconstr Surg 2001;107(01):73-79

7 Askari M, Fisher C, Weniger FG, Bidic S, Lee WP. Anticoagulation therapy in microsurgery: a review. J Hand Surg Am 2006;31(05): 836-846

8 Maeda M, Fukui A, Tamai S, Mizumoto S, Inada Y. Continuous local intra-arterial infusion of antithrombotic agents for replantation (comparison with intravenous infusion). Br J Plast Surg 1991;44 (07):520-525

9 Atiyeh BS, Hashim HA, Hamdan AM, Moucharafieh RS. Local recombinant tissue plasminogen activator (rt-PA) thrombolytic therapy in microvascular surgery. Microsurgery 1999;19(06):265-271

10 Lin CH, Aydyn N, Lin YT, Hsu CT, Lin CH, Yeh JT. Hand and finger replantation after protracted ischemia (more than 24 hours). Ann Plast Surg 2010;64(03):286-290 